\title{
LAGRANGE INTERPOLATION AND FINITE ELEMENT SUPERCONVERGENCE
}

\author{
BO LI
}

\begin{abstract}
We consider the finite element approximation of the Laplacian operator with the homogeneous Dirichlet boundary condition, and study the corresponding Lagrange interpolation in the context of finite element superconvergence. For $d$ dimensional $Q_{k}$-type elements with $d \geq 1$ and $k \geq 1$, we prove that the interpolation points must be the Lobatto points if the Lagrange interpolation and the finite element solution are superclose in $H^{1}$ norm. For $d$-dimensional $P_{k}$-type elements, we consider the standard Lagrange interpolation - the Lagrange interpolation with interpolation points being the principle lattice points of simplicial elements. We prove for $d \geq 2$ and $k \geq d+1$ that such interpolation and the finite element solution are not superclose in both $H^{1}$ and $L^{2}$ norms, and that not all such interpolation points are superconvergence points for the finite element approximation.
\end{abstract}

\section{INTRODUCTION}

Consider the boundary value problem

$$
\left\{\begin{aligned}
\mathcal{L} u=f & \text { in } \Omega, \\
u=0 & \text { on } \partial \Omega,
\end{aligned}\right.
$$

where $\Omega \subset \mathbb{R}^{d}$ is a bounded domain with a Lipschitz continuous boundary $\partial \Omega, d \geq 1$, $f \in L^{2}(\Omega)$, and $\mathcal{L}: H^{2}(\Omega) \rightarrow L^{2}(\Omega)$ is a second order, linear, self-adjoint, elliptic differential operator. Let $u \in H_{0}^{1}(\Omega)$ be its unique weak solution, defined by

$$
A(u, v)=(f, v) \quad \forall v \in H_{0}^{1}(\Omega),
$$

where $A: H_{0}^{1}(\Omega) \times H_{0}^{1}(\Omega) \rightarrow \mathbb{R}$ is the bilinear, symmetric, continuous, and coercive form associated with $(1.1)$, and $(\cdot, \cdot)$ denotes the inner product of $L^{2}(\Omega)$. Let $\left\{\tau_{h}\right\}$ be a family of finite element meshes of the domain $\Omega$ with the mesh size $h \rightarrow 0$. Fix an integer $k \geq 1$. For each $h$, let $S_{k}^{h}(\Omega) \subset H^{1}(\Omega) \cap C(\bar{\Omega})$ be the corresponding finite element space such that $\left.\left.S_{k}^{h}(\Omega)\right|_{T} \supseteq P_{k}\right|_{T}$ for all $T \in \tau_{h}$, where $P_{k}$ is the set of all polynomials of degree $\leq k$. Let $\stackrel{\circ}{S}_{k}^{h}(\Omega)=S_{k}^{h}(\Omega) \cap H_{0}^{1}(\Omega)$. Let $u_{h} \in \stackrel{\circ}{S}_{k}^{h}(\Omega)$ be the finite element solution, defined by

$$
A\left(u_{h}, v_{h}\right)=\left(f, v_{h}\right) \quad \forall v_{h} \in \stackrel{\circ}{S}_{k}^{h}(\Omega) .
$$

Date: June 12, 2003.

2000 Mathematics Subject Classification. 65N30.

Key words and phrases. finite element, Lagrange interpolation, superconvergence.

This work was partially supported by the NSF through grant DMS-0072958. 
Finally, let $I_{h}: C(\bar{\Omega}) \rightarrow S_{k}^{h}(\Omega)$ denote the corresponding Lagrange interpolation operator. The following estimate

$$
h^{-1}\left\|I_{h} u-u_{h}\right\|_{L^{2}(\Omega)}+\left\|I_{h} u-u_{h}\right\|_{H^{1}(\Omega)} \leq C h^{k}
$$

is standard, provided that the weak solution $u \in H_{0}^{1}(\Omega) \cap C(\bar{\Omega})$ is smooth enough and the underlying meshes are quasi-uniform $[3,9]$. Here and below, we use the letter $C$ to denote a generic, positive constant that is independent of the mesh size $h$.

The estimate (1.2) is in general optimal. However, in some cases, it can be improved. This means that $I_{h} u$ and $u_{h}$ can be superclose. More precisely, we say that the Lagrange interpolation $I_{h} u$ and the finite element solution $u_{h}$ are superclose in $H^{1}$ norm, if

$$
\left\|I_{h} u-u_{h}\right\|_{H^{1}(\Omega)}=o\left(h^{k}\right) \quad \text { as } h \rightarrow 0 .
$$

We also say that $I_{h} u$ and $u_{h}$ are superclose in $H^{1}$ norm by order (at least) $\sigma>0$, if

$$
\left\|I_{h} u-u_{h}\right\|_{H^{1}(\Omega)} \leq C h^{k+\sigma} .
$$

The following result gives a different expression of the closeness between $I_{h} u$ and $u_{h}$ in $H^{1}$ norm. It is trivially true, and we omit its proof.

Lemma 1.1. If the exact solution $u \in H_{0}^{1}(\Omega) \cap C(\bar{\Omega})$, then

$$
\gamma\left\|I_{h} u-u_{h}\right\|_{H^{1}(\Omega)} \leq \sup _{v_{h} \in S_{k}^{h}(\Omega), v_{h} \neq 0} \frac{\left|A\left(u-I_{h} u, v_{h}\right)\right|}{\left\|v_{h}\right\|_{H^{1}(\Omega)}} \leq M\left\|I_{h} u-u_{h}\right\|_{H^{1}(\Omega)},
$$

where $\gamma>0$ and $M>0$ are the two constants in the conditions of coercivity and continuity, respectively, of the bilinear form $A: H_{0}^{1}(\Omega) \times H_{0}^{1}(\Omega) \rightarrow \mathbb{R}$,

$$
A(v, v) \geq \gamma\|v\|_{H^{1}(\Omega)}^{2} \quad \forall v \in H_{0}^{1}(\Omega)
$$

and

$$
|A(v, w)| \leq M\|v\|_{H^{1}(\Omega)}\|w\|_{H^{1}(\Omega)} \quad \forall v, w \in H_{0}^{1}(\Omega) .
$$

The supercloseness between the Lagrange interpolation and the finite element solution is closely related to the superconvergence of the finite element solution to the exact solution. In fact, if (1.3) holds true, then one can easily obtain the following estimate of gradient superconvergence

$$
\left[\frac{1}{h^{d}} \sum_{z \in \mathcal{Z}_{h}(\Omega)}\left|\nabla u(z)-\bar{\nabla} u_{h}(z)\right|^{2}\right]^{1 / 2} \leq C h^{k+\min (\sigma, 1)},
$$

where $\mathcal{Z}_{h}(\Omega)$ is the set of superconvergence points for the gradient of the Lagrange interpolation and $\bar{\nabla}$ is some kind of average of the gradient [8]. In some cases, one can obtain a higher order estimate

$$
\left|A\left(u-I_{h} u, v_{h}\right)\right| \leq C h^{k+\sigma}\left\|v_{h}\right\|_{W^{1, p}(\Omega)} \quad \forall v_{h} \in \stackrel{\circ}{S}_{k}^{h}(\Omega)
$$

for some $\sigma>0$ and $p \in[1, \infty)$. This, together with delicate estimates of a discrete Green's function substituting $v_{h}$ in the inequality in (1.4), can lead to pointwise finite 
element superconvergence estimates $[1,5,26,28]$. By Lemma 1.1, the estimate (1.4) is equivalent to the supercloseness estimate (1.3), if $p=2$.

In this work, we study the supercloseness between the Lagrange interpolation and the finite element solution. Our main results are as follows.

1. For $d$-dimensional $Q_{k}$-type (tensor product) elements with $d \geq 1$ and $k \geq 1$, the interpolation points must be the Lobatto points if the Lagrange interpolation and the finite element solution are superclose in $H^{1}$ norm, cf. Theorem 2.1.

2. For $d$-dimensional $P_{k}$-type (simplicial) elements with $d \geq 2$ and $k \geq d+1$, the standard Lagrange interpolation - the Lagrange interpolation with its interpolation points being the principle lattice points of simplicial elements - and the finite element solution are not superclose in $H^{1}$ norm, cf. Theorem 4.1.

3. For $d$-dimensional $P_{k}$-type elements with $d \geq 2$ and $k \geq d+1$, not all the standard Lagrange interpolation points are superconvergence points for the finite element solution, cf. Corollary 4.1.

For $d$-dimensional $Q_{k}$-type elements with $d \geq 1$ and $k \geq 2$, the finite element solution is superconvergent by one order to the exact solution at the Lobatto points $[4,10,16,18,24,25]$. This implies that the Lagrange interpolation associated with the Lobatto points and the finite element solution are superclose by one order in $H^{1}$ norm. Here, we prove the converse under the assumption that they are only superclose, but not necessary superclose by any order, in $H^{1}$ norm.

For simplicial finite elements, the Lagrange interpolation points can not be arbitrarily distributed in general. With good meshes, the standard Lagrange interpolation and the finite element solution are in fact superclose in $H^{1}$ norm by one order for two-dimensional $P_{1}$ and $P_{2}$ elements and for three-dimensional $P_{1}$ element $[1,5-7,12-14,17,19,21,22,26-30]$. Recently, similar results have been obtained for any $d$-dimensional, linear, simplicial finite elements with a uniform mesh [2]. But, it is still open in general whether or not such supercloseness remains for $d$-dimensional $P_{k}$-type elements with $d \geq 3$ and $2 \leq k \leq d$.

The proof of those known results relies on lucky cancellation of inter-element boundary integrals in delicate estimates of the integral form $A\left(u-I_{h} u, v_{h}\right)$ for $v_{h} \in \stackrel{\circ}{S}_{k}^{h}(\Omega)$. However, such cancellation seems to be impossible if there exists an element-wise, bubble-like test function $v_{h} \in \stackrel{\circ}{S}_{k}^{h}(\Omega)$ that vanishes on the boundary of each element. Such a function exists if and only if there exists an interior node in each of the simplicial elements. This turns out to be true if and only if $k \geq d+1$ for $d$-dimensional $P_{k}$-type elements. Constructing a bubble-like test function to avoid any possible cancellation was the original approach in our early work [20] to show the the nonsupercloseness for two-dimensional $P_{3}$ element. Here, we extend such an approach to a general case which is more complicated due to the higher space dimension and higher polynomial degree.

In proving the non-supercloseness of the Lagrange interpolation to the finite element solution for the general $d$-dimensional $P_{k}$-type finite elements with $k \geq d+1$, we choose the underlying domain to be the unit $d$-dimensional simplex. This allows us to have a 
polynomial of degree exactly $k+1$ as the solution of the underlying Poisson equation with the homogeneous Dirichlet boundary condition. In addition, we construct a special family of quasi-uniform finite element meshes consisting of enough elements that are scaled translations of the unit simplex. Such meshes are uniform for $d=2$ but non-uniform for $d \geq 3$. Calculations based on such meshes are much simplified. With our approach, it is possible to consider a uniform family of finite element meshes of the $d$-dimensional unit cube, and construct similar but more complicated solutions. Undoubtedly, however, the calculations will be more involved.

In Section 2, we study the optimal Lagrange interpolation points for $Q_{k}$-type elements. In Section 3, we construct a quasi-uniform family of simplicial finite element meshes of a $d$-dimensional domain for $d \geq 2$. With such meshes, we study in Section 4 the standard Lagrange interpolation for $P_{k}$-type elements. Finally, in Section 5, we prove some auxiliary lemmas.

\section{Optimal Lagrange interpolation points For $Q_{k}$-Type Finite ELEMENTS}

Consider the boundary value problem

$$
\left\{\begin{aligned}
-\Delta u=f & \text { in } \Omega, \\
u=0 & \text { on } \partial \Omega,
\end{aligned}\right.
$$

where $f \in L^{2}(\Omega)$ and $\Omega=\prod_{m=1}^{d}\left(a_{m}, b_{m}\right) \subset \mathbb{R}^{d}$ is a $d$-dimensional rectangular parallelepiped with $d \geq 1$ and $-\infty<a_{m}<b_{m}<\infty$ for all $m=1, \cdots, d$. The associated bilinear form $A: H_{0}^{1}(\Omega) \times H_{0}^{1}(\Omega) \rightarrow \mathbb{R}$ is defined by

$$
A(v, w)=(\nabla v, \nabla w) \quad \forall v, w \in H_{0}^{1}(\Omega) .
$$

It is symmetric, continuous, and coercive. The weak solution $u \in H_{0}^{1}(\Omega)$ of the boundary value problem (2.1) is defined by

$$
A(u, v)=(f, v) \quad \forall v \in H_{0}^{1}(\Omega) .
$$

Let $\left\{\tau_{h}\right\}$ be a family of quasi-uniform rectangular meshes covering $\Omega$ with the mesh size $h \rightarrow 0$. We denote a typical mesh by

$$
\tau_{h}=\left\{\prod_{m=1}^{d}\left[x_{m, j_{m}-1}, x_{m, j_{m}}\right]: j_{m}=1, \cdots, n_{m}, m=1, \cdots, d\right\},
$$

where $x_{m, j_{m}}=a_{m}+j_{m} h_{m}$ for $j_{m}=0, \cdots, n_{m}, h_{m}=\left(b_{m}-a_{m}\right) / n_{m}, n_{m} \geq 1$ is an integer for each $m=1, \cdots, d$, and $h=\max _{1 \leq m \leq d} h_{m}$. For an integer $k \geq 1$, let $S_{k}^{h}(\Omega) \subset H^{1}(\Omega)$ denote the $Q_{k}$-type finite element space corresponding to the mesh $\tau_{h}$, i.e., the restriction $\left.S_{k}^{h}(\Omega)\right|_{R}$ is exactly $\left.Q_{k}\right|_{R}$ for each element $R \in \tau_{h}$, where

$$
Q_{k}=\operatorname{span}\left\{x_{1}^{\alpha_{1}} \cdots x_{d}^{\alpha_{d}}: \alpha_{1}, \cdots, \alpha_{d} \text { are nonnegative integers, } \alpha_{1}+\cdots+\alpha_{d}=k\right\} \text {. }
$$

Let $\stackrel{\circ}{S}_{k}^{h}(\Omega)=S_{k}^{h}(\Omega) \cap H_{0}^{1}(\Omega)$. The finite element solution $u_{h} \in \stackrel{\circ}{S}_{k}^{h}(\Omega)$ is defined by

$$
A\left(u_{h}, v_{h}\right)=\left(f, v_{h}\right) \quad \forall v_{h} \in \stackrel{\circ}{S}_{k}^{h}(\Omega) .
$$


For each integer $m$ with $1 \leq m \leq d$, let $\xi_{m}^{(0)}, \cdots, \xi_{m}^{(k)}$ be $k+1$ distinct real numbers satisfying

$$
-1=\xi_{m}^{(0)}<\cdots<\xi_{m}^{(k)}=1 .
$$

We call all the points $\left(\xi_{1}^{\left(i_{1}\right)}, \cdots, \xi_{d}^{\left(i_{d}\right)}\right)\left(i_{m}=0, \cdots, k, m=1, \cdots, d\right)$ the reference interpolation points. We define the Lagrange interpolation points on each element $\prod_{m=1}^{d}\left[x_{m, j_{m}-1}, x_{m, j_{m}}\right] \in \tau_{h}\left(1 \leq j_{m} \leq n_{m}, 1 \leq m \leq d\right)$ by

$$
x_{m, j_{m}}^{(i)}=\frac{h_{m} \xi_{m}^{(i)}+x_{m, j_{m}-1}+x_{m, j_{m}}}{2}, \quad i=0, \cdots, k, m=1, \cdots, d .
$$

Finally, we denote by $I_{h}: C(\bar{\Omega}) \rightarrow S_{k}^{h}(\Omega)$ the Lagrange interpolation operator associated with these interpolation points.

Recall that the Jacobi polynomials $P_{n}^{(1,1)}(n=0,1, \cdots)$ are orthogonal polynomials on the interval $[-1,1]$ with the weight $\rho(\xi)=1-\xi^{2}$, normalized by $P_{n}^{(1,1)}(1)=n+1$ [23]. The Rodrigues' formula for $P_{n}^{(1,1)}$ is

$$
P_{n}^{(1,1)}(\xi)=\frac{(-1)^{n}}{2^{n} n !\left(1-\xi^{2}\right)}\left(\frac{d}{d \xi}\right)^{n}\left[\left(1-\xi^{2}\right)^{n+1}\right] .
$$

For each $n \geq 1, P_{n}^{(1,1)}$ has exactly $n$ distinct roots in $(-1,1)$, called Lobatto points (associated with $n$ ). Recall also that the Legendre polynomials are orthogonal polynomials on the interval $[-1,1]$ with the weight $\rho(\xi)=1[23]$. They are given by

$$
L_{n}(\xi)=\frac{1}{2^{n} n !}\left(\frac{d}{d \xi}\right)^{n}\left[\left(1-\xi^{2}\right)^{n}\right], \quad n=0,1, \cdots .
$$

It is easy to show that $\left\{L_{n}^{\prime}(\xi)\right\}_{n=1}^{\infty}$ is also a sequence of orthogonal polynomials on $[-1,1]$ with the weight $\rho(\xi)=1-\xi^{2}$. Consequently, $P_{n}^{(1,1)}$ and $L_{n+1}^{\prime}$ differ only by a nonzero constant. For $n \geq 2$, the Lobatto points associated with $n-1$ are thus the roots of $L_{n}^{\prime}(\xi)$ in $(-1,1)$. However, for convenience, we shall call in what follows all the $n-1$ distinct roots of $L_{n}^{\prime}(\xi)$ in $(-1,1)$, together with \pm 1 , the Lobatto points of order $n$. (In fact, \pm 1 are often included in a Lobatto quadrature [11].) We call a point in $\mathbb{R}^{d}$ a $d$-dimensional Lobatto point of order $n$, if each of its $d$ coordinates is a one-dimensional Lobatto point of order $n$. Obviously, there are $(n+1)^{d} d$-dimensional Lobatto points of order $n$.

Together with what is known, the following result implies for $Q_{k}$-type finite elements with $k \geq 2$ that the Lagrange interpolation is superclose to the finite element solution in $H^{1}$ norm if and only if all the interpolation points are the Lobatto points.

Theorem 2.1. Suppose that

$$
\left\|I_{h} u-u_{h}\right\|_{H^{1}(\Omega)}=o\left(h^{k}\right) \quad \text { as } h \rightarrow 0,
$$

whenever the solution $u \in H_{0}^{1}(\Omega)$ is smooth enough. Then, all the reference interpolation points $\left(\xi_{1}^{\left(i_{1}\right)}, \cdots, \xi_{d}^{\left(i_{d}\right)}\right)\left(0 \leq i_{m} \leq k, m=1, \cdots, d\right)$ must be the d-dimensional Lobatto points of order $k$. 
Proof. For $k=1$, the reference interpolation points are always Lobatto points by our definition. So, we assume that $k \geq 2$. We shall show for each $m(1 \leq m \leq d)$ that $\xi_{m}^{(0)}, \cdots, \xi_{m}^{(k)}$ are indeed the $k+1$ one-dimensional Lobatto points of order $k$.

Fix an index $m$ with $1 \leq m \leq d$. Define $u \in H_{0}^{1}(\Omega)$ by

$$
u(x)=\left(x_{m}-\frac{a_{m}+b_{m}}{2}\right)^{k-1} \prod_{l=1}^{d}\left(x_{l}-a_{l}\right)\left(x_{l}-b_{l}\right), \quad x=\left(x_{1}, \cdots, x_{d}\right) \in \bar{\Omega} .
$$

Note that $u$ depends on $m$. Define accordingly $f(x)=-\Delta u(x)$ for all $x \in \Omega$. Obviously, $f \in L^{2}(\Omega)$, and $u \in C^{\infty}(\bar{\Omega})$ solves the boundary value problem (2.1).

For each integer $s: 0 \leq s \leq k-2$, define $v_{s}:\left[a_{m}, b_{m}\right] \rightarrow \mathbb{R}$ by

$$
\begin{aligned}
& v_{s}\left(x_{m}\right)=\phi_{s}\left(\frac{2 x_{m}-x_{m, j_{m}-1}-x_{m, j_{m}}}{h_{m}}\right), \\
& \forall x_{m} \in\left[x_{m, j_{m}-1}, x_{m, j_{m}}\right], j_{m}=1, \cdots, n_{m},
\end{aligned}
$$

where the function $\phi_{s}:[-1,1] \rightarrow \mathbb{R}$ is defined by

$$
\phi_{s}(\xi)=\xi^{s+2}-\frac{1}{2}(1+\xi)-\frac{1}{2}(-1)^{s}(1-\xi), \quad \xi \in[-1,1] .
$$

It is easy to see that

$$
\phi_{s}^{\prime \prime}(\xi)=(s+1)(s+2) \xi^{s} \quad \text { and } \quad \phi_{s}(-1)=\phi_{s}(1)=0 .
$$

Hence, $v_{s}$ is a continuous piecewise polynomial of degree $s+2 \leq k$, vanishing at all the points $x_{m, j_{m}}\left(j_{m}=0, \cdots, n_{m}\right)$. Define $v_{h}: \bar{\Omega} \rightarrow \mathbb{R}$ for the case $d=1$ by $v_{h}\left(x_{1}\right)=v_{s}\left(x_{1}\right)$ for all $x_{1} \in \bar{\Omega}=\left[a_{1}, b_{1}\right]$, and for the case $d \geq 2$ by

$$
v_{h}(x)=v_{s}\left(x_{m}\right) W_{m}\left(x^{\prime}\right) \quad \forall x \in \bar{\Omega},
$$

where

$$
\begin{aligned}
W_{m}\left(x^{\prime}\right) & =\prod_{l=1, l \neq m}^{d}\left(x_{l}-a_{l}\right)\left(x_{l}-b_{l}\right) \quad \forall x^{\prime} \in \Omega^{\prime}, \\
x^{\prime} & =\left(x_{1}, \cdots, x_{m-1}, x_{m+1}, \cdots, x_{d}\right), \\
\Omega^{\prime} & =\prod_{l=1, l \neq m}^{d}\left(a_{l}, b_{l}\right) .
\end{aligned}
$$

Note that $v_{h}$ depends on $m$ and that $v_{h} \in \stackrel{\circ}{S}_{k}^{h}(\Omega)$.

Assume that $d \geq 2$ temporarily. Let $R=\prod_{l=1}^{d}\left[x_{l, j_{l}-1}, x_{l, j_{l}}\right] \in \tau_{h}$ be an arbitrary element, where $1 \leq j_{l} \leq n_{l}$ and $1 \leq l \leq d$. The function

$$
u(x)-W_{m}\left(x^{\prime}\right) \prod_{i=0}^{k}\left(x_{m}-x_{m, j_{m}}^{(i)}\right)
$$


is in $\left.Q_{k}\right|_{R}$, and agrees with $u$ on all the interpolation points in $R$. Hence, this function is exactly the Lagrange interpolation of $u$ on the element $R$. Thus, we have

$$
\left(u-I_{h} u\right)(x)=W_{m}\left(x^{\prime}\right) \prod_{i=0}^{k}\left(x_{m}-x_{m, j_{m}}^{(i)}\right) \quad \forall x \in R .
$$

Let $R^{\prime}=\prod_{l=1, l \neq m}^{d}\left[x_{l, j_{l}-1}, x_{l, j_{l}}\right]$. Let $\nabla^{\prime}$ denote the gradient operator with respect to $x^{\prime}$. Applying integration by parts and using the change of variable

$$
\xi_{m}=\frac{2 x_{m}-x_{m, j_{m}-1}-x_{m, j_{m}}}{h_{m}}
$$

from $x_{m} \in\left[x_{m, j_{m}-1}, x_{m, j_{m}}\right]$ to $\xi_{m} \in[-1,1]$, we obtain that

$$
\begin{aligned}
\int_{R} \nabla & \left.u-I_{h} u\right)(x) \cdot \nabla v_{h}(x) d x \\
= & \int_{R}\left[\nabla^{\prime}\left(u-I_{h} u\right)(x) \cdot \nabla^{\prime} v_{h}(x)+\frac{\partial}{\partial x_{m}}\left(u-I_{h} u\right)(x) \cdot \frac{\partial}{\partial x_{m}} v_{h}(x)\right] d x \\
= & \int_{R}\left|\nabla^{\prime} W_{m}\left(x^{\prime}\right)\right|^{2} v_{s}\left(x_{m}\right) \prod_{i=0}^{k}\left(x_{m}-x_{m, j_{m}}^{(i)}\right) d x \\
& +\int_{R}\left[W_{m}\left(x^{\prime}\right)\right]^{2}\left[\frac{d}{d x_{m}} \prod_{i=0}^{k}\left(x_{m}-x_{m, j_{m}}^{(i)}\right)\right] v_{s}^{\prime}\left(x_{m}\right) d x \\
= & \int_{R^{\prime}}\left|\nabla^{\prime} W_{m}\left(x^{\prime}\right)\right|^{2} d x^{\prime} \int_{x_{m, j_{m}-1}}^{x_{m, j_{m}}} v_{s}\left(x_{m}\right) \prod_{i=0}^{k}\left(x_{m}-x_{m, j_{m}}^{(i)}\right) d x_{m} \\
& -\int_{R^{\prime}}\left[W_{m}\left(x^{\prime}\right)\right]^{2} d x^{\prime} \int_{x_{m, j_{m}-1}}^{x_{m, j_{m}}} v_{s}^{\prime \prime}\left(x_{m}\right) \prod_{i=0}^{k}\left(x_{m}-x_{m, j_{m}}^{(i)}\right) d x_{m} \\
= & \left(\frac{h_{m}}{2}\right)^{k+2}\left\|\nabla^{\prime} W_{m}\right\|_{L^{2}\left(R^{\prime}\right)}^{2} \int_{-1}^{1} \phi_{s}\left(\xi_{m}\right) \prod_{i=0}^{k}\left(\xi_{m}-\xi_{m}^{(i)}\right) d \xi_{m} \\
& -\left(\frac{h_{m}}{2}\right)^{k}\left\|W_{m}\right\|_{L^{2}\left(R^{\prime}\right)}^{2} \int_{-1}^{1} \phi_{s}^{\prime \prime}\left(\xi_{m}\right) \prod_{i=0}^{k}\left(\xi_{m}-\xi_{m}^{(i)}\right) d \xi_{m} .
\end{aligned}
$$

Consequently, we have by the fact $n_{m}=\left(b_{m}-a_{m}\right) / h_{m}$ that

$$
\begin{aligned}
& A\left(u-I_{h} u, v_{h}\right)=\sum_{R \in \tau_{h}} \int_{R} \nabla\left(u-I_{h} u\right)(x) \cdot \nabla v_{h}(x) d x \\
& =\frac{\left(b_{m}-a_{m}\right)}{2^{k+2}} h_{m}^{k+1}\left\|\nabla^{\prime} W_{m}\right\|_{L^{2}\left(\Omega^{\prime}\right)}^{2} \int_{-1}^{1} \phi_{s}\left(\xi_{m}\right) \prod_{i=0}^{k}\left(\xi_{m}-\xi_{m}^{(i)}\right) d \xi_{m}
\end{aligned}
$$




$$
-\frac{\left(b_{m}-a_{m}\right)(s+1)(s+2)}{2^{k}} h_{m}^{k-1}\left\|W_{m}\right\|_{L^{2}\left(\Omega^{\prime}\right)}^{2} \int_{-1}^{1} \xi_{m}^{s} \prod_{i=0}^{k}\left(\xi_{m}-\xi_{m}^{(i)}\right) d \xi_{m} .
$$

Similarly, we obtain that

$$
\begin{aligned}
\left\|v_{h}\right\|_{H^{1}(\Omega)}^{2}= & \int_{\Omega}\left[\left|v_{h}(x)\right|^{2}+\left|\nabla v_{h}(x)\right|^{2}\right] d x \\
= & \left\|W_{m}\right\|_{H^{1}\left(\Omega^{\prime}\right)}^{2} \sum_{j_{m}=1}^{n_{m}} \int_{x_{m, j_{m}-1}}^{x_{m, j_{m}}}\left|v_{s}\left(x_{m}\right)\right|^{2} d x_{m} \\
& \quad+\left\|W_{m}\right\|_{L^{2}\left(\Omega^{\prime}\right)}^{2} \sum_{j_{m}=1}^{n_{m}} \int_{x_{m, j_{m}-1}}^{x_{m, j_{m}}}\left|v_{s}^{\prime}\left(x_{m}\right)\right|^{2} d x_{m} \\
= & \frac{\left(b_{m}-a_{m}\right)}{2}\left\|W_{m}\right\|_{H^{1}\left(\Omega^{\prime}\right)}^{2} \int_{-1}^{1}\left|\phi_{s}\left(\xi_{m}\right)\right|^{2} d \xi_{m} \\
& \quad+\frac{2\left(b_{m}-a_{m}\right)}{h_{m}^{2}}\left\|W_{m}\right\|_{L^{2}\left(\Omega^{\prime}\right)}^{2} \int_{-1}^{1}\left|\phi_{s}^{\prime}\left(\xi_{m}\right)\right|^{2} d \xi_{m},
\end{aligned}
$$

leading to

$$
\left\|v_{h}\right\|_{H^{1}(\Omega)} \leq \sqrt{\frac{\left(b_{m}-a_{m}\right)\left[4+\left(b_{m}-a_{m}\right)^{2}\right]}{2}}\left\|\phi_{s}\right\|_{H^{1}(-1,1)}\left\|W_{m}\right\|_{H^{1}\left(\Omega^{\prime}\right)} h_{m}^{-1} .
$$

Therefore, we infer from (2.3) and (2.4) that

$$
\frac{\left|A\left(u-I_{h} u, v_{h}\right)\right|}{\left\|v_{h}\right\|_{H^{1}(\Omega)}} \geq \alpha_{1} h_{m}^{k}\left|\int_{-1}^{1} \xi_{m}^{s} \prod_{i=0}^{k}\left(\xi_{m}-\xi_{m}^{(i)}\right) d \xi_{m}\right|-\alpha_{2} h_{m}^{k+2},
$$

where

$$
\alpha_{1}=\sqrt{\frac{2\left(b_{m}-a_{m}\right)}{4+\left(b_{m}-a_{m}\right)^{2}}} \frac{(s+1)(s+2)\left\|W_{m}\right\|_{L^{2}\left(\Omega^{\prime}\right)}^{2}}{2^{k}\left\|\phi_{s}\right\|_{H^{1}(-1,1)}\left\|W_{m}\right\|_{H^{1}\left(\Omega^{\prime}\right)}}>0
$$

and

$$
\begin{gathered}
\alpha_{2}=\sqrt{\frac{2\left(b_{m}-a_{m}\right)}{4+\left(b_{m}-a_{m}\right)^{2}} \frac{\left\|\nabla^{\prime} W_{m}\right\|_{L^{2}\left(\Omega^{\prime}\right)}^{2}}{2^{k+2}\left\|\phi_{s}\right\|_{H^{1}(-1,1)}\left\|W_{m}\right\|_{H^{1}\left(\Omega^{\prime}\right)}}} \\
\cdot\left|\int_{-1}^{1} \phi_{s}\left(\xi_{m}\right) \prod_{i=0}^{k}\left(\xi_{m}-\xi_{m}^{(i)}\right) d \xi_{m}\right| \geq 0
\end{gathered}
$$

are constants independent of $h$.

Assume now $d=1$. By a similar but simpler argument, we obtain that

$$
A\left(u-I_{h} u, v_{h}\right)=-\frac{\left(b_{1}-a_{1}\right)(s+1)(s+2)}{2^{k}} h_{1}^{k-1} \int_{-1}^{1} \xi_{1}^{s} \prod_{i=0}^{k}\left(\xi_{1}-\xi_{1}^{(i)}\right) d \xi_{1}
$$


and

$$
\left\|v_{h}\right\|_{H^{1}(\Omega)}^{2}=\frac{b_{1}-a_{1}}{2} \int_{-1}^{1}\left[\left|\phi_{s}\left(\xi_{1}\right)\right|^{2}+\frac{4}{h_{1}^{2}}\left|\phi_{s}^{\prime}\left(\xi_{1}\right)\right|^{2}\right] d \xi_{1} .
$$

Therefore,

$$
\left\|v_{h}\right\|_{H^{1}(\Omega)} \leq \sqrt{\frac{\left(b_{1}-a_{1}\right)\left[4+\left(b_{1}-a_{1}\right)^{2}\right]}{2}}\left\|\phi_{s}\right\|_{L^{2}(-1,1)} h_{1}^{-1}
$$

and

$$
\frac{\left|A\left(u-I_{h} u, v_{h}\right)\right|}{\left\|v_{h}\right\|_{H^{1}(\Omega)}} \geq \beta h_{1}^{k}\left|\int_{-1}^{1} \xi_{1}^{s} \prod_{i=0}^{k}\left(\xi_{1}-\xi_{1}^{(i)}\right) d \xi_{1}\right|
$$

where

$$
\beta=\sqrt{\frac{2\left(b_{1}-a_{1}\right)}{4+\left(b_{1}-a_{1}\right)^{2}}} \frac{(s+1)(s+2)}{2^{k}\left\|\phi_{s}\right\|_{L^{2}(-1,1)}}>0
$$

is a constant independent of $h$.

It now follows from Lemma 1.1, (2.2), (2.5), (2.6), and the quasi-uniformity of the meshes that

$$
\int_{-1}^{1} \xi_{m}^{s} \prod_{i=0}^{k}\left(\xi_{m}-\xi_{m}^{(i)}\right) d \xi_{m}=0, \quad s=0, \cdots, k-2 .
$$

The polynomial $\prod_{i=1}^{k-1}\left(\xi-\xi_{m}^{(i)}\right)$ of degree $k-1$ is thus orthogonal to all the polynomials in $P_{k-2}$ on $[-1,1]$ with the weight $\left(\xi-\xi_{m}^{(0)}\right)\left(\xi-\xi_{m}^{(k)}\right)=\xi^{2}-1$. Hence, it differs from the Jacobi polynomial $P_{k-1}^{(1,1)}$ only by a nonzero constant. Consequently, all the points $\xi_{m}^{(0)}, \cdots, \xi_{m}^{(k)}$ are the $k+1$ one-dimensional Lobatto points of order $k$.

\section{A COnstruction of $d$-Dimensional Simplicial Finite ElEMENT MEShes}

We now let $d \geq 2$ be an integer and $\Omega \subset \mathbb{R}^{d}$ the open unit simplex

$$
\Omega=\left\{\left(x_{1}, \cdots, x_{d}\right) \in \mathbb{R}^{d}: x_{i}>0, i=1, \cdots, d, \sum_{i=1}^{d} x_{i}<1\right\} .
$$

We shall construct a quasi-uniform family of simplicial finite element meshes $\left\{\tau_{h}\right\}$ of $\Omega$ such that there are $O\left(h^{-d}\right)$ elements in $\tau_{h}$ that are translations of a single $d$ dimensional simplex $\sigma_{d}^{-1} h \bar{\Omega}=\left\{\sigma_{d}^{-1} h x: x \in \bar{\Omega}\right\}$, where $\sigma_{d}>0$ is a constant depending only on $d$ and $h$ is the mesh size of $\tau_{h}$.

For $d=2$, the mesh $\tau_{h}$ can be defined by three families of parallel lines $x_{1}=i / n$, $x_{2}=j / n$, and $x_{1}+x_{2}=l / n$, where $n \geq 1$ is an integer and $i, j, l=0, \cdots, n$. This is a uniform mesh with mesh size $h=\sqrt{2} / n$. Obviously, there are $O\left(h^{-2}\right)$ elements of the mesh that are translations of the single 2-dimensional simplex $\sigma_{2}^{-1} h \bar{\Omega}$ with $\sigma_{2}=\sqrt{2}$.

For $d \geq 3$, we construct in three steps a simplicial finite element mesh of $\Omega$ with the designed properties. First, we triangulate the reference unit cube into simplexes. Second, we construct a simplicial finite element mesh of the unit cube by cutting it into many small cubes, triangulating them by affine mappings from the triangulated 
reference unit cube, and gluing them together. Third, we cut the meshed unit cube by the plane $\sum_{i=1}^{d} x_{i}=1$ to define a simplicial finite element mesh of $\Omega$.

Step 1. Take the closed unit cube $C_{d}=[0,1]^{d} \subset \mathbb{R}^{d}$ as the reference cube and denote by $\xi=\left(\xi_{1}, \cdots, \xi_{d}\right)$ a generic point in $C_{d}$. Define

$$
B_{d}^{l}=\left\{\xi \in C_{d}: l-1 \leq \sum_{i=1}^{d} \xi_{i} \leq l\right\}, \quad l=1, \cdots, d .
$$

Obviously,

$$
\cup_{l=1}^{d} B_{d}^{l}=C_{d} \quad \text { and } \quad \operatorname{int}\left(B_{d}^{j}\right) \cap \operatorname{int}\left(B_{d}^{l}\right)=\emptyset \quad \text { if } j \neq l .
$$

Notice that $B_{d}^{1}=S_{d}$, where

$$
S_{d}=\left\{\left(\xi_{1}, \cdots, \xi_{d}\right) \in \mathbb{R}^{d}: \xi_{i} \geq 0, i=1, \cdots, d, \sum_{i=1}^{d} \xi_{i} \leq 1\right\}
$$

is the reference unit simplex in $\mathbb{R}^{d}$, and both $B_{d}^{1}$ and $B_{d}^{d}$ are simplexes. But $B_{d}^{l}$ is not a simplex if $1<l<d$. This is because that the number of vertices in a $d$ dimensional simplex is $d+1$. However, since all the vertices $\left(\xi_{1}, \cdots, \xi_{d}\right)\left(\xi_{i}=0\right.$ or 1 , $i=1, \cdots, d)$ of $C_{d}$ lie in the planes $\sum_{i=1}^{d} \xi_{i}=j(j=0, \cdots, d)$, the number of vertices of $C_{d}$ contained in $B_{d}^{l}$ is the same as that contained in the planes $\sum_{i=1}^{d} \xi_{i}=l-1$ and $\sum_{i=1}^{d} \xi_{i}=l$. This number is

$$
\left(\begin{array}{c}
d \\
l-1
\end{array}\right)+\left(\begin{array}{l}
d \\
l
\end{array}\right)=\left(\begin{array}{c}
d+1 \\
l
\end{array}\right)>d+1
$$

since $1<l<d$.

We now triangulate all the polygons $B_{d}^{l}(l=2, \cdots d-1)$ into simplexes so that, together with $B_{d}^{1}$ and $B_{d}^{d}$, these simplexes can form a simplicial triangulation of $C_{d}$. It suffices to triangulate the boundary of each $B_{d}^{l}$ into $(d-1)$-dimensional simplexes determined by a set of vertices, and then connect the barycenter of $B_{d}^{l}$ to these vertices. The boundary of $B_{d}^{l}$ for each $l$ with $2 \leq l \leq d-1$ is the union of two types of $(d-1)$-dimensional polygons

$$
P_{d-1}^{m}=\left\{\xi \in C_{d}: \sum_{i=1}^{d} \xi_{i}=m\right\}, \quad m=l-1, l,
$$

and

$$
F_{d-1}^{l, j, m}=B_{d}^{l} \cap\left\{\xi \in C_{d}: \xi_{j}=m\right\}, \quad j=1, \cdots, d, m=0,1 .
$$

Consider a first type $(d-1)$-dimensional polygon $P_{d-1}^{m}(1 \leq m \leq d-1)$. If $m=1$ or $d-1$, then $P_{d-1}^{m}$ is already a $(d-1)$-dimensional simplex. Suppose $2 \leq m \leq$ $d-2$. To triangulate $P_{d-1}^{m}$ into $(d-1)$-dimensional simplexes, we again need only to triangulate the boundary of $P_{d-1}^{m}$ into $(d-2)$-dimensional simplexes and then connect 
the barycenter of $P_{d-1}^{m}$ to all the vertices in such a $(d-2)$-dimensional simplicial triangulation. The boundary of $P_{d-1}^{m}$ is the union of the following sets:

$$
P_{d-1}^{m} \cap\left\{\xi \in C_{d}: \xi_{j}=0\right\} \quad \text { and } \quad P_{d-1}^{m} \cap\left\{\xi \in C_{d}: \xi_{j}=1\right\}, \quad j=1, \cdots, d .
$$

Each of these sets is either already a $(d-2)$-dimensional simplex (if $m=2$ and $\xi_{j}=1$ ) or still a first type polygon but of one-dimension lower. For $d=3$, both $P_{2}^{1}$ and $P_{2}^{2}$ are already 2-dimensional simplexes. Therefore, we conclude by induction that, for $d \geq 3$ in general, all the first type $(d-1)$-dimensional polygons $P_{d-1}^{m} \subset \mathbb{R}^{d-1}$ $(m=1, \cdots, d-1)$ can be triangulated into $(d-1)$-dimensional simplexes. Notice that

$$
\begin{gathered}
P_{d-1}^{m} \cap\left\{\xi \in C_{d}: \xi_{j}=1\right\}=e_{j}+P_{d-1}^{m-1} \cap\left\{\xi \in C_{d}: \xi_{j}=0\right\}, \\
j=1, \cdots, d, m=2, \cdots, d-1,
\end{gathered}
$$

where $e_{j} \in \mathbb{R}^{d}$ is the point with the $j$-th coordinate 1 and all others 0 .

Consider now a second type $(d-1)$-dimensional polygon $F_{d-1}^{l, j, m}(2 \leq l \leq d-1,1 \leq$ $j \leq d, m=0,1)$. If $l=2$ and $m=1$, or $l=d-1$ and $m=0$, then $F_{d-1}^{l, j, m}$ is already a

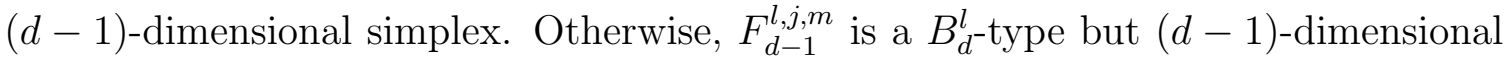
polygon, cf. (3.2). For $d=3$, there are altogether six of such 2-dimensional polygons $F_{2}^{2, j, m}(j=1,2,3, m=0,1)$. All of them are 2-dimensional simplexes. So, by induction, the second type $(d-1)$-dimensional polygons $F_{d-1}^{l, j, m}$ with $d \geq 3$ can all be triangulated into $(d-1)$-dimensional simplexes. Notice that

$$
F_{d-1}^{l, j, 1}=e_{j}+F_{d-1}^{l-1, j, 0}, \quad l=2, \cdots, d-1, j=1, \cdots, d .
$$

Finally, for each $l \in\{2, \cdots, d-1\}$, we connect the barycenter of polygon $B_{d}^{l}$ to all the vertices in the constructed $(d-1)$-dimensional triangulation of the boundary of $B_{d}^{l}$. This results in a triangulation of $B_{d}^{l}$ into $d$-dimensional simplexes. All these simplexes in the triangulation of $B_{d}^{l}$ for $l=2, \cdots, d-1$, together with the simplexes $B_{d}^{1}=S_{d}$ and $B_{d}^{d}$, form a simplicial triangulation of the unit cube $C_{d}$.

By the construction, cf. (3.2), (3.5), and (3.6), the simplicial triangulation of the reference unit cube $C_{d}$ satisfies the following properties.

1. The unit simplex $S_{d}$ is a simplicial element of the triangulation.

2. Triangulation symmetry: for each integer $i$ with $1 \leq i \leq d$, the restriction of the simplicial triangulation of the reference unit cube $C_{d}$ on the two faces $\xi_{i}=0$ and $\xi_{i}=1$ results in the same $(d-1)$-dimensional simplicial triangulation of the $(d-1)$-dimensional unit cube

$$
\begin{aligned}
C_{d-1}^{i}=\{ & \left(\xi_{1}, \cdots, \xi_{i-1}, \xi_{i+1}, \cdots, \xi_{d}\right) \in \mathbb{R}^{d-1}: \\
& \left.0 \leq \xi_{j} \leq 1, j=1, \cdots, i-1, i+1, \cdots, d\right\} .
\end{aligned}
$$

3. For any integer $j$ with $1 \leq j \leq d$, the plane $\sum_{i=1}^{d} \xi_{i}=j$ does not intersect the interior of any simplicial element of the triangulation. 
Step 2. Fix an integer $n \geq 1$ and use planes $x_{i}=j / n(i=1, \cdots, d, j=0, \cdots, n)$ to cut the unit cube $[0,1]^{d}$ into $n^{d}$ small cubes. Let $c_{d}=\prod_{i=1}^{d}\left[x_{i}^{0}, x_{i}^{0}+1 / n\right]$ denote a typical such small cube. Define $G: C_{d} \rightarrow c_{d}$ by $G(\xi)=(1 / n) \xi+x^{0}$ for all $\xi \in C_{d}$, where $x^{0}=\left(x_{1}^{0}, \cdots, x_{d}^{0}\right)$. Obviously, it is a one-to-one and onto, orientation preserving, and affine mapping from the reference unit cube $C_{d}$ to the small cube $c_{d}$. Therefore, together with the constructed simplicial triangulation of the reference unit cube $C_{d}$, the mapping $G: C_{d} \rightarrow c_{d}$ defines a simplicial triangulation of the small cube $c_{d}$. By the arbitrariness of $c_{d}$ and the property of triangulation symmetry of the simplicial triangulation of the reference unit cube, we have in fact constructed a simplicial finite element mesh of the unit cube $[0,1]^{d}$. The mesh size is $h=\sigma_{d} / n$, where $\sigma_{d}$ is the maximum of diameters of simplexes in the constructed triangulation of the reference unit cube $C_{d}$.

The constructed simplicial finite element mesh of the unit cube $[0,1]^{d}$ satisfies the following properties.

1. Each small cube $c_{d}=\prod_{i=1}^{d}\left[x_{i}^{0}, x_{i}^{0}+1 / n\right]$ contains one simplicial element

$$
s_{d}=\left\{\left(x_{1}, \cdots, x_{d}\right) \in c_{d}: \sum_{i=1}^{d}\left(x_{i}-x_{i}^{0}\right) \leq \frac{1}{n}\right\},
$$

which is a translation of the simplex $(1 / n) S_{d}=\sigma_{d}^{-1} h S_{d}$. Thus, there are $n^{d}=$ $\left(\sigma_{d} / h\right)^{d}$ simplicial elements in the mesh that are translations of the single simplex $\sigma_{d}^{-1} h S_{d}$

2. The plane $\sum_{i=1}^{d} x_{i}=1$ does not intersect the interior of any simplicial element.

3 . If the plane $\sum_{i=1}^{d} x_{i}=1$ intersects the interior of a small cube $c_{d}=\prod_{i=1}^{d}\left[x_{i}^{0}, x_{i}^{0}+\right.$ $1 / n]$, then the simplex $s_{d}$ must be in $\bar{\Omega}$.

The first property follows from the first property in Step 1 and our construction of the finite element mesh of the unit cube $[0,1]^{d}$. To show the other two properties, we consider a typical small cube $c_{d}=\prod_{i=1}^{d}\left[x_{i}^{0}, x_{i}^{0}+1 / n\right]$. After the change of variables $\xi=$ $n\left(x-x^{0}\right)$, where $x^{0}=\left(x_{1}^{0}, \cdots, x_{d}^{0}\right)$, the cube $c_{d}$ is transformed into the reference unit cube $C_{d}$ and the plane $\sum_{i=1}^{d} x_{i}=1$ into $\sum_{i=1}^{d} \xi_{i}=j_{0}$, where $j_{0}=n\left(1-\sum_{i=1}^{d} x_{i}^{0}\right)$. Notice that $j_{0}$ is an integer, since all $n x_{i}^{0}(i=1, \ldots, d)$ are integers. If $j_{0} \notin\{1, \cdots, d-$ $1\}$, then the plane $\sum_{i=1}^{d} x_{i}=1$ does not cut the interior of the small cube $c_{d}$, by our triangulation of the reference unit cube $C_{d}$, cf. (3.2) and (3.3). Otherwise, $j_{0} \in\{1, \cdots, d-1\}$. In this case, the plane $\sum_{i=1}^{d} x_{i}=1$ cuts the interior of the small cube $c_{d}$ but not the interior of any simplicial element, by the last property stated in Step 1. Moreover, $\sum_{i=1}^{d}\left(x_{i}^{0}+1 / n\right) \leq 1$, since $j_{0} \geq 1$. Hence, the small simplex $s_{d}$ is contained in $\bar{\Omega}$.

Step 3. Cut the constructed simplicial finite element mesh of the unit cube by the plane $\sum_{i=1}^{d} x_{i}=1$. By the second property in Step 2, we have constructed a simplicial finite element mesh $\tau_{h}$ of the domain $\Omega$. Since the $d$-dimensional volume of $\Omega$ is $1 / d$ ! and that of each small cube is $1 / n^{d}$, it follows from the first property in 
Step 2, this mesh $\tau_{h}$ contains $O\left(h^{-d}\right)$ simplicial elements which are translations of the single simplex $\sigma_{d}^{-1} h S_{d}=\sigma_{d}^{-1} h \bar{\Omega}$.

Letting $n=1, \cdots$, we then obtain a family of simplicial finite element meshes $\left\{\tau_{h}\right\}$. Since we only use a single reference triangulation to construct each mesh, the family of meshes $\left\{\tau_{h}\right\}$ are quasi-uniform.

We summarize our results in the following theorem.

Theorem 3.1. Let $d \geq 2$ be an integer and $\Omega \subset \mathbb{R}^{d}$ the d-dimensional open unit simplex. Then, there exist a quasi-uniform family of simplicial finite element meshes $\left\{\tau_{h}\right\}$ of $\Omega$ such that each of the meshes $\tau_{h}$ contains $O\left(h^{-d}\right)$ simplicial elements which are translations of the single simplex $\sigma_{d}^{-1} h S_{d}$, where $h$ is the mesh size of $\tau_{h}$ and $\sigma_{d}>0$ a constant depending only on the dimension $d$.

\section{On the Standard LAGRANGE INTERPolation FOR $d$-DIMENSIONAL $P_{k}$-TYPE} FINITE ELEMENTS

Let $d \geq 2$ be an integer and $\Omega \subset \mathbb{R}^{d}$ the open unit simplex defined in (3.1). Let $k$ be an integer such that $k \geq d+1$. Define $u: \bar{\Omega} \rightarrow \mathbb{R}$ by

$$
u(x)=\left(1-\sum_{i=1}^{d} x_{i}\right)^{k+1-d} \prod_{i=1}^{d} x_{i} \quad \forall x=\left(x_{1}, \cdots, x_{d}\right) \in \bar{\Omega} .
$$

Define also $f(x)=-\Delta u(x)$ for $x \in \Omega$. Obviously, $u \in H_{0}^{1}(\Omega) \cap C^{\infty}(\bar{\Omega})$ solves the boundary value problem

$$
\left\{\begin{aligned}
-\Delta u=f & \text { in } \Omega, \\
u=0 & \text { on } \partial \Omega .
\end{aligned}\right.
$$

Equivalently, $u \in H_{0}^{1}(\Omega)$ is the weak solution, defined by

$$
A(u, v)=(f, v) \quad \forall v \in H_{0}^{1}(\Omega),
$$

where $A: H_{0}^{1}(\Omega) \times H_{0}^{1}(\Omega) \rightarrow \mathbb{R}$, defined by

$$
A(v, w)=(\nabla v, \nabla w) \quad \forall v, w \in H_{0}^{1}(\Omega),
$$

is the bilinear form associated with the boundary value problem (4.1), It is symmetric, continuous, and coercive.

Let $n \geq 1$ be an integer and $\tau_{h}$ the corresponding simplicial finite element mesh of $\Omega$ constructed in Section 3. Let $S_{k}^{h}(\Omega) \subset H^{1}(\Omega)$ denote the $P_{k}$-type finite element space corresponding to the mesh $\tau_{h}$, i.e., the restriction $\left.S_{k}^{h}(\Omega)\right|_{T}$ is exactly $\left.P_{k}\right|_{T}$ for each element $T \in \tau_{h}$. Let $\stackrel{\circ}{S}_{k}^{h}(\Omega)=S_{k}^{h}(\Omega) \cap H_{0}^{1}(\Omega)$. The finite element solution $u_{h} \in \stackrel{\circ}{S}_{k}^{h}(\Omega)$ is defined by

$$
A\left(u_{h}, v_{h}\right)=\left(f, v_{h}\right) \quad \forall v_{h} \in \stackrel{\circ}{S}_{k}^{h}(\Omega) .
$$

Finally, denote by $I_{h}: C(\bar{\Omega}) \rightarrow S_{k}^{h}(\Omega)$ the standard Lagrange interpolation whose interpolation points are the principle lattice points of all simplex elements of $\tau_{h}[3,9]$. 
Theorem 4.1. Let $d$ and $k$ be integers such that $d \geq 2$ and $k \geq d+1$. With the quasi-uniform family of simplicial finite element meshes constructed in Section 3, we have that

$$
\left\|I_{h} u-u_{h}\right\|_{H^{1}(\Omega)} \geq \zeta_{d, k} h^{k}
$$

where $\zeta_{d, k}>0$ is a constant depending only on $d$ and $k$.

Proof. We shall call $T \in \tau_{h}$ a corner simplicial element if

$$
T=\left\{\left(x_{1}, \cdots, x_{d}\right) \in R^{d}: x_{i}-\frac{j_{i}}{n} \geq 0, i=1, \cdots, d, \sum_{i=1}^{d}\left(x_{i}-\frac{j_{i}}{n}\right) \leq \frac{1}{n}\right\}
$$

for some integers $j_{i}$ with $0 \leq j_{i} \leq n-1, i=1, \ldots, d$. For such an element, we denote its $d+1$ vertices by

$$
\begin{aligned}
& x^{(0)}=\left(\frac{j_{1}}{n}, \cdots, \frac{j_{d}}{n}\right), \\
& x^{(i)}=\left(\frac{j_{1}}{n}, \cdots, \frac{j_{i-1}}{n}, \frac{j_{i}+1}{n}, \frac{j_{i+1}}{n}, \cdots, \frac{j_{d}}{n}\right), \quad i=1, \cdots, d .
\end{aligned}
$$

For each $x \in T$, let $\lambda_{i}(x)(i=0, \cdots, d)$ be the barycentric coordinates of $x$ defined by $\left.\lambda_{i} \in P_{1}\right|_{T}$, and $\lambda_{i}\left(x^{(j)}\right)=1$ if $i=j$ and 0 if $i \neq j$. Explicitly,

$$
\begin{array}{ll}
\lambda_{i}(x)=n\left(x_{i}-\frac{j_{i}}{n}\right), & i=1, \cdots, d, x=\left(x_{1}, \cdots, x_{d}\right) \in T, \\
\lambda_{0}(x)=1-\sum_{i=1}^{d} \lambda_{i}(x), & x \in T .
\end{array}
$$

Define $\psi_{T}: T \rightarrow \mathbb{R}$ by

$$
\psi_{T}(x)=\left[\prod_{i=1}^{d} \lambda_{i}(x)\right] \prod_{j=0}^{k-d-1}\left[\lambda_{0}(x)-\frac{j}{k}\right] \quad \forall x \in T .
$$

We claim that $\psi_{T}$ differs only by a nonzero constant from the local shape function associated with the nodal point

$$
\tilde{x}=\left(\frac{j_{1}}{n}+\frac{1}{n k}, \cdots, \frac{j_{d}}{n}+\frac{1}{n k}\right) \in T
$$

whose barycentric coordinate is

$$
\left(\lambda_{0}(\tilde{x}), \lambda_{1}(\tilde{x}), \cdots, \lambda_{d}(\tilde{x})\right)=\left(\frac{k-d}{k}, \frac{1}{k}, \cdots, \frac{1}{k}\right) \in \mathbb{R}^{d+1} .
$$

In fact, $\left.\psi_{T} \in P_{k}\right|_{T}$. Moreover, any nodal point $x \in T$ has the barycentric coordinates $\lambda_{i}(x)=m_{i} / k$ for some integer $m_{i}$ with $0 \leq m_{i} \leq k, i=0, \cdots, d$, and $m_{0}=$ $k-\sum_{i=1}^{d} m_{i}$. If $0 \leq m_{0} \leq k-d-1$, then $\psi_{T}(x)=0$. If $k-d+1 \leq m_{0} \leq k$, then at least one $m_{i}=0(1 \leq i \leq d)$, implying that $\psi_{T}(x)=0$. If $m_{0}=k-d$, 
then $\sum_{i=1}^{d} m_{i}=d$. In this case, if for some $i(1 \leq i \leq d) m_{i}=0$, then $\psi_{T}(x)=0$. Otherwise, all $m_{i}=1(i=1, \cdots, d)$, and $x=\tilde{x}$. But, $\psi_{T}(\tilde{x})=(k-d) ! / k^{k}>0$.

Denoting by $I_{T}:\left.C(T) \rightarrow P_{k}\right|_{T}$ the local Lagrange interpolation operator on $T$ - the restriction of $I_{h}: C(\bar{\Omega}) \rightarrow S_{h}^{k}(\Omega)$ onto $C(T)$, we then have

$$
\left(I_{T}\left(\lambda_{0} \psi_{T}\right)\right)(x)=\lambda_{0}(\tilde{x}) \psi_{T}(x)=\frac{k-d}{k} \psi_{T}(x) \quad \forall x \in T .
$$

Consequently, since on $T, u(x)-n^{-k-1} \lambda_{0}(x) \psi_{T}(x)$ is a polynomial of degree $\leq k$, and $I_{T}:\left.C(T) \rightarrow P_{k}\right|_{T}$ is a projection on $\left.P_{k}\right|_{T}$, we have that

$$
\begin{aligned}
u(x)-\left(I_{T} u\right)(x) & =n^{-k-1}\left[\left(\lambda_{0} \psi_{T}\right)(x)-\left(I_{T}\left(\lambda_{0} \psi_{T}\right)\right)(x)\right] \\
& =n^{-k-1}\left[\lambda_{0}(x)-\frac{k-d}{k}\right] \psi_{T}(x) \\
& =n^{-k-1}\left[\prod_{i=1}^{d} \lambda_{i}(x)\right] \prod_{j=0}^{k-d}\left[\lambda_{0}(x)-\frac{j}{k}\right] \quad \forall x \in T .
\end{aligned}
$$

We now define $\left.\left.v_{T} \in P_{d+1}\right|_{T} \subseteq P_{k}\right|_{T}$ by $v_{T}(x)=\prod_{i=0}^{d} \lambda_{i}(x)$ for all $x \in T$. By a simple calculation, we have that

$$
\Delta v_{T}(x)=-2 n^{2} \sum_{i=1}^{d} \prod_{j=1, j \neq i}^{d} \lambda_{j}(x) \quad \forall x \in T .
$$

Moreover, using the change of variables $\xi_{i}=\lambda_{i}(x)(i=1, \cdots, d)$ from $x \in T$ to $\xi \in S_{d}$, we obtain that

$$
\left\|v_{T}\right\|_{H^{1}(T)}^{2}=n^{-d} \int_{S_{d}} \prod_{i=0}^{d} \xi_{i}^{2} d \xi+n^{2-d} \int_{S_{d}}\left|\nabla_{\xi}\left(\prod_{i=0}^{d} \xi_{i}\right)\right|^{2} d \xi
$$

where $\xi_{0}=1-\sum_{i=1}^{d} \xi_{i}$ and $\nabla_{\xi}$ is the gradient with respect to $\xi$.

By (4.3), $u-I_{T} u$ vanishes on the boundary of $T$. Therefore, by integration by parts, $(4.3),(4.4)$, and the change of variables $\xi_{i}=\lambda_{i}(x)(i=1, \cdots, d)$ from $x \in T$ to $\xi \in S_{d}$, we get that

$$
\begin{aligned}
\int_{T} \nabla & \left(u-I_{T} u\right)(x) \cdot \nabla v_{T}(x) d x \\
& =-\int_{T}\left(u-I_{T} u\right)(x) \Delta v_{T}(x) d x \\
& =2 n^{1-k} \int_{T}\left[\prod_{i=1}^{d} \lambda_{i}(x)\right]\left\{\prod_{j=0}^{k-d}\left[\lambda_{0}(x)-\frac{j}{k}\right]\right\} \sum_{i=1}^{d} \prod_{j=1, j \neq i}^{d} \lambda_{j}(x) d x \\
= & 2 n^{1-k-d} \int_{S_{d}}\left(\prod_{i=1}^{d} \xi_{i}\right)\left[\prod_{j=0}^{k-d}\left(\xi_{0}-\frac{j}{k}\right)\right] \sum_{i=1}^{d} \prod_{j=1, j \neq i}^{d} \xi_{j} d \xi
\end{aligned}
$$


Denote by $\tau_{h}^{\prime}$ the collection of all the corner simplicial elements in $\tau_{h}$. Define $v_{h}: \bar{\Omega} \rightarrow \mathbb{R}$ by $v_{h}=0$ on all elements in $\tau_{h} \backslash \tau_{h}^{\prime}$ and $v_{h}=v_{T}$ on any element $T \in \tau_{h}^{\prime}$. We have that $v_{h} \in \stackrel{\circ}{S}_{k}^{h}(\Omega)$, since for each $T \in \tau_{h}^{\prime},\left.v_{T} \in P_{k}\right|_{T}$ vanishes on the boundary of $T$. Moreover, we have by (4.5) that

$$
\left\|v_{h}\right\|_{H^{1}(\Omega)}^{2}=n^{2-d}\left|\tau_{h}^{\prime}\right|\left[n^{-2} \int_{S_{d}} \prod_{i=0}^{d} \xi_{i}^{2} d \xi+\int_{S_{d}}\left|\nabla_{\xi}\left(\prod_{i=1}^{d} \xi_{i}\right)\right|^{2} d \xi\right],
$$

where $\left|\tau_{h}^{\prime}\right|$ is the number of elements in $\tau_{h}^{\prime}$, and by (4.6) that

$$
A\left(u-I_{h} u, v_{h}\right)=\sum_{T \in \tau_{h}^{\prime}} \int_{T} \nabla\left(u-I_{T} u\right)(x) \cdot \nabla v_{T}(x) d x=2 \mu_{d, k}\left|\tau_{h}^{\prime}\right| n^{1-k-d},
$$

where

$$
\mu_{d, k}=\int_{S_{d}}\left(\prod_{i=1}^{d} \xi_{i}\right)\left[\prod_{j=0}^{k-d}\left(\xi_{0}-\frac{j}{k}\right)\right] \sum_{i=1}^{d} \prod_{j=1, j \neq i}^{d} \xi_{j} d \xi
$$

is a constant depending only on $d$ and $k$. Consequently,

$$
\frac{\left|A\left(u-I_{h} u, v_{h}\right)\right|}{\left\|v_{h}\right\|_{\left.H^{(} \Omega\right)}} \geq\left(\frac{2\left|\mu_{d, k}\right|}{\sqrt{\nu_{d}}}\right)\left|\tau_{h}^{\prime}\right|^{1 / 2} n^{-k-d / 2},
$$

where

$$
\nu_{d}=\int_{S_{d}}\left[\prod_{i=0}^{d} \xi_{i}^{2}+\left|\nabla_{\xi}\left(\prod_{i=1}^{d} \xi_{i}\right)\right|^{2}\right] d \xi>0
$$

is a constant depending only on $d$.

It follows from the construction of the mesh $\tau_{h}$ in Section 3 that $h=\sigma_{d} / n$ and $\left|\tau_{h}^{\prime}\right| \geq \kappa_{d} h^{-d}$ for some constants $\sigma_{d}>0$ and $\kappa_{d}>0$ that depend only on $d$. Moreover, $\mu_{d, k} \neq 0$ by Lemma 5.1 below. Therefore, the desired inequality (4.2) follows from (4.7) and Lemma 1.1 with

$$
\zeta_{d, k}=\sqrt{\frac{4 \kappa_{d} \mu_{d, k}^{2}}{\sigma_{d}^{2 k+d} \nu_{d, k}}}>0,
$$

where we use the fact that the constant $M$ in the continuity condition in Lemma 1.1 can be taken as 1 in the present case.

Corollary 4.1. Let $d$ and $k$ be integers such that $d \geq 2$ and $k \geq d+1$. With the quasi-uniform family of simplicial finite element meshes constructed in Section 3, we have that

$$
\max _{z \in \mathcal{N}_{h}}\left|u(z)-u_{h}(z)\right| \geq \theta_{d, k} h^{k+1},
$$

where $\mathcal{N}_{h}$ is the set of all the standard Lagrange interpolation points and $\theta_{d, k}>0$ is a constant depending only on $d$ and $k$. 
Proof. Notice that $\left(I_{h} u\right)(z)=u(z)$ for all $z \in \mathcal{N}_{h}$. Thus, if (4.8) were not true, then we would have

$$
\left\|I_{h} u-u_{h}\right\|_{L^{\infty}(\Omega)}=o\left(h^{k+1}\right) \quad \text { as } h \rightarrow 0 .
$$

This would lead to

$$
\left\|I_{h} u-u_{h}\right\|_{L^{2}(\Omega)}=o\left(h^{k+1}\right) \quad \text { as } h \rightarrow 0,
$$

and further to

$$
\left\|I_{h} u-u_{h}\right\|_{H^{1}(\Omega)}=o\left(h^{k}\right) \quad \text { as } h \rightarrow 0
$$

by an inverse estimate, contradicting the assertion of Theorem 4.1.

\section{Auxiliary Lemmas}

Lemma 5.1. We have for any integers $d$ and $k$ satisfying $d \geq 2$ and $k \geq d+1$ that

$$
\mu_{d, k}:=\int_{S_{d}}\left(\prod_{i=1}^{d} \xi_{i}\right)\left[\prod_{j=0}^{k-d}\left(\xi_{0}-\frac{j}{k}\right)\right] \sum_{i=1}^{d} \prod_{j=1, j \neq i}^{d} \xi_{j} d \xi \neq 0,
$$

where $S_{d}$ is the d-dimensional unit simplex defined in (3.4) and $\xi_{0}=1-\sum_{i=1}^{d} \xi_{i}$.

Proof. By the symmetry about the variables $\xi_{1}, \cdots, \xi_{d}$, and the change of variables $\eta_{i}=\xi_{i}(i=1, \cdots, d-1)$ and $\eta_{d}=1-\sum_{i=1}^{d} \xi_{i}$, we have

$$
\begin{aligned}
\mu_{d, k}= & d \int_{S_{d}}\left(\prod_{i=1}^{d} \xi_{i}\right)\left[\prod_{j=0}^{k-d}\left(\xi_{0}-\frac{j}{k}\right)\right] \prod_{i=1}^{d-1} \xi_{i} d \xi \\
= & d \int_{S_{d}}\left(\prod_{i=1}^{d-1} \eta_{i}^{2}\right)\left(1-\sum_{i=1}^{d} \eta_{i}\right) \prod_{j=0}^{k-d}\left(\eta_{d}-\frac{j}{k}\right) d \eta \\
= & d \int_{0}^{1} d \eta_{d} \int_{0}^{1-\eta_{d}} d \eta_{d-1} \cdots \int_{0}^{1-\sum_{i=j+1}^{d} \eta_{i}} d \eta_{j} \cdots \int_{0}^{1-\sum_{i=2}^{d} \eta_{i}} d \eta_{1} \\
& \left(\prod_{i=1}^{d-1} \eta_{i}^{2}\right)\left(1-\sum_{i=1}^{d} \eta_{i}\right) \prod_{j=0}^{k-d}\left(\eta_{d}-\frac{j}{k}\right) .
\end{aligned}
$$

Set

$$
E_{1}=\int_{0}^{1-\sum_{i=2}^{d} \eta_{i}} \eta_{1}^{2}\left(1-\sum_{i=1}^{d} \eta_{i}\right) d \eta_{1}
$$

and

$$
E_{j}=\int_{0}^{1-\sum_{i=j+1}^{d} \eta_{i}} \eta_{j}^{2} E_{j-1} d \eta_{j} \quad \text { for } j=2, \cdots, d-1 .
$$

By an argument of induction on $j(1 \leq j \leq d-1)$ using the expression

$$
\eta_{j}^{2}=\left[\left(1-\sum_{i=j}^{d} \eta_{i}\right)-\left(1-\sum_{i=j+1}^{d} \eta_{i}\right)\right]^{2}
$$




$$
=\left(1-\sum_{i=j}^{d} \eta_{i}\right)^{2}-2\left(1-\sum_{i=j}^{d} \eta_{i}\right)\left(1-\sum_{i=j+1}^{d} \eta_{i}\right)+\left(1-\sum_{i=j+1}^{d} \eta_{i}\right)^{2}
$$

we obtain that

$$
E_{j}=\left[\prod_{i=1}^{j} \frac{2}{(3 i-1)(3 i)(3 i+1)}\right]\left(1-\sum_{i=j+1}^{d} \eta_{i}\right)^{3 j+1}, \quad j=1, \cdots, d-1 .
$$

It then follows from (5.1) that

$$
\begin{aligned}
\mu_{d, k} & =d \int_{0}^{1} E_{d-1} \prod_{j=0}^{k-d}\left(\eta_{d}-\frac{j}{k}\right) d \eta_{d} \\
& =d\left[\prod_{i=1}^{d-1} \frac{2}{(3 i-1)(3 i)(3 i+1)}\right] \int_{0}^{1}\left(1-\eta_{d}\right)^{3 d-2} \prod_{j=0}^{k-d}\left(\eta_{d}-\frac{j}{k}\right) d \eta_{d} .
\end{aligned}
$$

This is a nonzero constant by Lemma 5.2 below.

Lemma 5.2. We have for any integers $d$ and $k$ satisfying $d \geq 2$ and $k \geq d+1$ that

$$
J_{d, k}:=\int_{0}^{1}(1-t)^{3 d-2} \prod_{i=0}^{k-d}\left(t-\frac{i}{k}\right) d t \begin{cases}>0 & \text { if } k-d \text { is even, } \\ <0 & \text { if } k-d \text { is odd. }\end{cases}
$$

Proof. Denote $q=k-d \geq 1$ and $\omega_{l}(t)=\prod_{i=0}^{l}\left(t-t_{i}\right)$ for any integer $l \geq 0$, where $t_{r}=r / k$ for any real $r$.

Case 1: $q=k-d$ is even. Let $\Omega_{l}(t)=\int_{0}^{t} \omega_{l}(s) d s$. By [15] (Lemma 4 on page 309), we have $\Omega_{q}(t)>0$ for all $t \in\left(0, t_{q}\right)$ and $\Omega_{q}\left(t_{q}\right)=0$. Moreover, for $t_{q}<t \leq 1$, we have

$$
\Omega_{q}(t)=\Omega_{q}\left(t_{q}\right)+\int_{t_{q}}^{t} \omega_{q}(s) d s=\int_{t_{q}}^{t} \omega_{q}(s) d s>0,
$$

since $\omega_{q}(s)>0$ for all $s \geq t_{q}$. Therefore, we obtain by integration by parts that

$$
\begin{aligned}
J_{d, k} & =\int_{0}^{1}(1-t)^{3 d-2} \Omega_{q}^{\prime}(t) d t=(3 d-2) \int_{0}^{1}(1-t)^{3 d-3} \Omega_{q}(t) d t \\
& =(3 d-2) \int_{0}^{t_{q}}(1-t)^{3 d-3} \Omega_{q}(t) d t+(3 d-2) \int_{t_{q}}^{1}(1-t)^{3 d-3} \Omega_{q}(t) d t>0 .
\end{aligned}
$$

Case 2: $q=k-d$ is odd. Direct calculations lead to

$$
J_{d, d+q}=-\frac{(d-1) \gamma_{q}(d)}{(d+q)^{q}} \prod_{i=-1}^{q}(3 d+i)^{-1}<0, \quad q=1,3,5,7,
$$

where

$$
\begin{aligned}
& \gamma_{1}(d)=1>0 \\
& \gamma_{3}(d)=12\left(4 d^{2}-d+3\right)>0
\end{aligned}
$$




$$
\begin{gathered}
\gamma_{5}(d)=6\left(1257 d^{4}+513 d^{3}+3031 d^{2}-225 d+2400\right)>0, \\
\gamma_{7}(d)=72\left(36163 d^{6}+83793 d^{5}+250663 d^{4}+144355 d^{3}\right. \\
\left.+410070 d^{2}+53452 d+352800\right)>0 .
\end{gathered}
$$

Therefore, we may and shall assume that $q=k-d \geq 9$. We have

$$
J_{d, k}=\int_{0}^{t_{q}}(1-t)^{3 d-2} \omega_{q}(t) d t+\int_{t_{q}}^{1}(1-t)^{3 d-2} \omega_{q}(t) d t=I_{d, k}+M_{d, k} .
$$

By straight forward calculations, we obtain that

$$
\begin{aligned}
M_{d, k} & :=\sum_{j=1}^{d} \int_{t_{q+j-1}}^{t_{q+j}}(1-t)^{3 d-2} \prod_{i=0}^{q}\left(t-t_{i}\right) d t \\
& <\sum_{j=1}^{d}\left[\prod_{i=0}^{q}\left(t_{q+j}-t_{i}\right)\right] \int_{t_{q+j-1}}^{t_{q+j}}(1-t)^{3 d-2} d t \\
& =\frac{1}{(3 d-1) k^{2 d+k}} \sum_{j=1}^{d}\left[\prod_{i=0}^{q}(i+j)\right]\left[(d-j+1)^{3 d-1}-(d-j)^{3 d-1}\right] \\
& =\frac{1}{(3 d-1) k^{2 d+k}}\left\{\left[\prod_{i=0}^{q}(1+i)\right] d^{3 d-1}\right] \\
& \left.=\frac{q+1}{(3 d-1) k^{2 d+k}} \sum_{j=0}^{d-1}\left[\prod_{i=1}^{q}(i+j+1)-\prod_{i=0}^{q}(i+j)\right](d-j)^{3 d-1}\right\}
\end{aligned}
$$

where in the third step we use the Abel summation identity

$$
\sum_{j=1}^{d} u_{j}\left(v_{j-1}-v_{j}\right)=u_{1} v_{0}-u_{d} v_{d}+\sum_{j=1}^{d-1}\left(u_{j+1}-u_{j}\right) v_{j}
$$

with $u_{j}=\prod_{i=0}^{q}(i+j)$ and $v_{j}=(d-j)^{3 d-1}$.

Since $q \geq 9$ is odd, we have by [15] (Lemma 2 on page 309) that $\omega_{q}(t)=\omega_{q}\left(t_{q}-t\right.$ ) for all $t \in\left[t_{q / 2}, t_{q}\right]$. Thus, by the change of variable $t_{q}-t \rightarrow s$ from $t \in\left[t_{q / 2}, t_{q}\right]$ to $s \in\left[0, t_{q / 2}\right]$, we get

$$
\begin{aligned}
I_{d, k} & :=\int_{0}^{t_{q / 2}}(1-t)^{3 d-2} \omega_{q}(t) d t+\int_{t_{q / 2}}^{t_{q}}(1-t)^{3 d-2} \omega_{q}\left(t_{q}-t\right) d t \\
& =\int_{0}^{t_{q / 2}}\left[(1-t)^{3 d-2}+\left(1-t_{q}+t\right)^{3 d-2}\right] \omega_{q}(t) d t
\end{aligned}
$$




$$
\begin{aligned}
& =\sum_{j=1}^{q_{0}} \int_{t_{2 j-2}}^{t_{2 j}}\left[(1-t)^{3 d-2}+\left(1-t_{q}+t\right)^{3 d-2}\right] \omega_{q}(t) d t \\
& \quad+\int_{t_{2 q_{0}}}^{t_{q / 2}}\left[(1-t)^{3 d-2}+\left(1-t_{q}+t\right)^{3 d-2}\right] \omega_{q}(t) d t \\
& =\sum_{j=1}^{q_{0}} H_{d, k, j}+G_{d, k},
\end{aligned}
$$

where

$$
q_{0}= \begin{cases}(q-3) / 4 & \text { if }(q-1) / 2 \text { is odd } \\ (q-1) / 4 & \text { if }(q-1) / 2 \text { is even }\end{cases}
$$

We show now that

$$
G_{d, k}:=\int_{t_{2 q_{0}}}^{t_{q / 2}}\left[(1-t)^{3 d-2}+\left(1-t_{q}+t\right)^{3 d-2}\right] \omega_{q}(t) d t<0 .
$$

If $(q-1) / 2$ is even, then for any $t \in\left(t_{2 q_{0}}, t_{q / 2}\right), \omega_{q}(t)$ has $2 q_{0}-1$ negative factors. Hence, it is negative. Thus (5.7) holds true. If $(q-1) / 2$ is odd, then for any $t \in\left(t_{2 q_{0}+1 / 2}, t_{2 q_{0}+1}\right), \omega_{q}(t)<0$, since it has $2 q_{0}+3$ negative factors. Hence, by the change of variable $t \rightarrow t-1 / k$ from $\left[t_{2 q_{0}+1}, t_{2 q_{0}+3 / 2}\right]$ to $\left[t_{2 q_{0}}, t_{2 q_{0}+1 / 2}\right]$, we obtain that

$$
\begin{aligned}
G_{d, k}= & \left(\int_{t_{2 q_{0}}}^{t_{2 q_{0}+1 / 2}}+\int_{t_{2 q_{0}+1 / 2}}^{t_{2 q_{0}+1}}+\int_{t_{2 q_{0}+1}}^{t_{2 q_{0}+3 / 2}}\right)\left[(1-t)^{3 d-2}+\left(1-t_{q}+t\right)^{3 d-2}\right] \omega_{q}(t) d t \\
< & \int_{t_{2 q_{0}}}^{t_{2 q_{0}+1 / 2}}\left[(1-t)^{3 d-2}+\left(1-t_{q}+t\right)^{3 d-2}\right] \omega_{q}(t) d t \\
& \quad+\int_{t_{2 q_{0}+1}}^{t_{2 q_{0}+3 / 2}}\left[(1-t)^{3 d-2}+\left(1-t_{q}+t\right)^{3 d-2}\right] \omega_{q}(t) d t \\
= & \int_{t_{2 q_{0}}}^{t_{2 q_{0}+1 / 2}}\left[(1-t)^{3 d-2}+\left(1-t_{q}+t\right)^{3 d-2}\right] \omega_{q}(t) d t \\
& \quad+\int_{t_{2 q_{0}}}^{t_{2 q_{0}+1 / 2}}\left[\left(1-\frac{1}{k}-t\right)^{3 d-2}+\left(1-t_{q}+\frac{1}{k}+t\right)^{3 d-2}\right] \omega_{q}\left(\frac{1}{k}+t\right) d t \\
= & -\int_{t_{2 q_{0}}}^{t_{2 q_{0}+1 / 2}} g_{d, k}(t) \omega_{q-1}(t) d t
\end{aligned}
$$

where

$$
g_{d, k}(t)=\left(t_{q}-t\right) f_{k, d}(t)-\left(t+\frac{1}{k}\right) f_{d, k}\left(t+\frac{1}{k}\right)
$$

and

$$
f_{d, k}(t)=(1-t)^{3 d-2}+\left(1-t_{q}+t\right)^{3 d-2}
$$


For $t \in\left(t_{2 q_{0}}, t_{2 q_{0}+1 / 2}\right)$, there are $2 q_{0}+2$ negative factors in $\omega_{q-1}(t)$. So, $\omega_{q-1}(t)>0$. Moreover, $f_{d, k}(t)>0$ and $f_{d, k}^{\prime}(t)<0$ for all $t \in\left(0, t_{q / 2}\right)$. Thus,

$$
g_{d, k}(t) \geq\left(t_{q}-t\right) f_{d, k}(t)-\left(t+\frac{1}{k}\right) f_{d, k}(t) \geq \frac{1}{k} f_{d, k}(t)>0 \quad \forall t \in\left(t_{2 q_{0}}, t_{2 q_{0}+1 / 2}\right) .
$$

Therefore, (5.7) also holds true.

Fix now $j \in\left\{1, \cdots, q_{0}\right\}$. By the change of variable $t \rightarrow t-1 / k$ from $\left[t_{2 j-1}, t_{2 j}\right]$ to $\left[t_{2 j-2}, t_{2 j-1}\right]$, we get that

$$
\begin{aligned}
H_{d, k, j} & :=\int_{t_{2 j-2}}^{t_{2 j}}\left[(1-t)^{3 d-2}+\left(1-t_{q}+t\right)^{3 d-2}\right] \omega_{q}(t) d t \\
& =\left(\int_{t_{2 j-2}}^{t_{2 j-1}}+\int_{t_{2 j-1}}^{t_{2 j}}\right)\left[(1-t)^{3 d-2}+\left(1-t_{q}+t\right)^{3 d-2}\right] \omega_{q}(t) d t \\
& =-\int_{t_{2 j-2}}^{t_{2 j-1}} g_{d, k}(t) \omega_{q-1}(t) d t
\end{aligned}
$$

where $g_{d, k}$ is defined in (5.8). For each $t \in\left(t_{2 j-2}, t_{2 j-1}\right), \omega_{q-1}(t)$ has $4 q_{0}-2 j+4$ negative factors. So, $\omega_{q-1}(t)>0$. Using the fact that $(-1)^{s} f_{d, k}^{(s)}(t)>0$ for all $t \in\left(0, t_{q / 2}\right)$ and $s=0,1,2$, where $f_{d, k}$ is defined in (5.9), we easily obtain that

$$
g_{d, k}(t) \geq\left(t_{q}-2 t-\frac{1}{k}\right) f_{d, k}\left(t+\frac{1}{k}\right) \geq \frac{4}{k} f_{d, k}\left(t+\frac{1}{k}\right)>0
$$

and

$$
\begin{aligned}
g_{d, k}^{\prime}(t) & =-\left[f_{d, k}(t)+f_{d, k}\left(t+\frac{1}{k}\right)\right]+\left(t_{q}-t\right) f_{d, k}^{\prime}(t)-\left(t+\frac{1}{k}\right) f_{d, k}^{\prime}\left(t+\frac{1}{k}\right) \\
& <\left(t_{q}-2 t-\frac{1}{k}\right) f_{d, k}^{\prime}\left(t+\frac{1}{k}\right) \\
& <0
\end{aligned}
$$

for all $t \in\left(t_{2 j-2}, t_{2 j-1}\right)$. Therefore,

$$
H_{d, k, j}<0, \quad j=1, \cdots, q_{0} .
$$

By (5.11), we have that

$$
g_{d, k}(t) \geq g_{d, k}\left(t_{1}\right)=t_{q-1} f_{d, k}\left(t_{1}\right)-t_{2} f\left(t_{2}\right)>t_{q-3} f_{d, k}\left(t_{1}\right)>0, \quad t_{0}<t<t_{1} .
$$

Consequently, by (5.6), (5.7), (5.10), (5.12), and the fact that $\omega_{q-1}(t)>0$ for $t \in$ $\left(t_{0}, t_{1}\right)$, we conclude that

$$
\begin{aligned}
I_{d, k} & <H_{d, k, 1} \\
& <-g_{d, k}\left(t_{1}\right) \int_{t_{0}}^{t_{1}} \omega_{q-1}(t) d t
\end{aligned}
$$




$$
\begin{aligned}
& <-t_{q-3} f_{d, k}\left(t_{1}\right) \prod_{i=2}^{q-1}\left(t_{i}-t_{1}\right) \int_{t_{0}}^{t_{1}} t\left(t_{1}-t\right) d t \\
& <-\frac{(q-3)(q-2) !}{6 k^{2 d+k}}(q+d-1)^{3 d-2} .
\end{aligned}
$$

It follows now from (5.4), (5.5), and (5.13) that we need only to show that

$$
\frac{6(q+1)}{(3 d-1)(q-3)} \sum_{j=0}^{d-1}\left[\prod_{i=1}^{q}(i+j)\right](d-j)^{3 d-2} \leq(q-2) !(q+d-1)^{3 d-2}
$$

for all the integers $d \geq 2$ and $q \geq 9$. For $d=2$, one can easily verify that this inequality holds true for all $q \geq 9$. Therefore, since

$$
\frac{6(q+1)}{(3 d-1)(q-3)}=\frac{2}{d}\left(1+\frac{1}{3 d-1}\right)\left(1+\frac{4}{q-3}\right) \leq \frac{4}{d}, \quad d \geq 3, q \geq 9,
$$

to complete the proof of the lemma, we need only to show that

$$
\sum_{j=0}^{d-1}\left[\prod_{i=1}^{q}(i+j)\right](d-j)^{3 d-2} \leq \frac{d}{4}(q-2) !(q+d-1)^{3 d-2}, \quad d \geq 3, q \geq 9 .
$$

For each index $j$ with $0 \leq j \leq d-1$, we have by the binomial formula that

$$
\begin{aligned}
& (q-2) !(q+d-1)^{3 d-2} \\
& =(q-2) ![(d-j)+(q+j-1)]^{3 d-2} \\
& \geq(q-2) ! \sum_{m=j}^{3 d-2}\left(\begin{array}{c}
3 d-2 \\
m
\end{array}\right)(d-j)^{3 d-2-m}(q+j-1)^{m} \\
& =\left[\prod_{i=1}^{q}(i+j)\right](d-j)^{3 d-2} \sum_{m=j}^{3 d-2} \frac{[(3 d-2) \cdots(3 d-1-m)](q+j-1)^{m}}{[(j+1) \cdots m][(q-1) \cdots(q+j)](d-j)^{m}} \\
& \geq\left[\prod_{i=1}^{q}(i+j)\right](d-j)^{3 d-2} \sum_{m=j}^{3 d-2} \frac{[(3 d-2) \cdots(3 d-1-m)](q+j-1)^{m-j-1}}{[(j+1) \cdots m](q+j)(d-j)^{m}} \\
& =\left[\prod_{i=1}^{q}(i+j)\right](d-j)^{3 d-2} S_{j},
\end{aligned}
$$

that is,

$$
(q-2) !(q+d-1)^{3 d-2} S_{j}^{-1} \geq\left[\prod_{i=1}^{q}(i+j)\right](d-j)^{3 d-2}, \quad j=0, \cdots, d-1,
$$

where

$$
S_{j}=\sum_{m=j}^{3 d-2} \frac{[(3 d-2) \cdots(3 d-1-m)](q+j-1)^{m-j-1}}{[(j+1) \cdots m](q+j)(d-j)^{m}}, \quad j=0, \cdots, d-1 .
$$


For $j=0$ and $j=1$, keeping only the term with $m=4$ and $m=5$ in the summation $S_{0}$ and $S_{1}$, respectively, and using the fact that $q \geq 9$, we get that

$$
\begin{aligned}
S_{0} & \geq \frac{[(3 d-2)(3 d-3)(3 d-4)(3 d-5)](q-1)^{3}}{24 q d^{4}} \\
& \geq \frac{[(2 d) \cdot(2 d) \cdot d \cdot d](q-1)^{3}}{24 q d^{4}} \\
& \geq 9
\end{aligned}
$$

and that

$$
\begin{aligned}
S_{1} & \geq \frac{[(3 d-2)(3 d-3)(3 d-4)(3 d-5)(3 d-6)] q^{3}}{120(q+1)(d-1)^{5}} \\
& \geq \frac{[3(d-1) \cdot 3(d-1) \cdot 2(d-1) \cdot 2(d-1) \cdot(d-1)] q^{3}}{120(q+1)(d-1)^{5}} \\
& \geq 21 .
\end{aligned}
$$

For $2 \leq j \leq d-1$, we get by keeping only the term with $m=\min (3 j-1, q+j-1)$ in the sum $S_{j}$ that

$$
\begin{aligned}
S_{j} & \geq \frac{[(3 d-2) \cdots(3 d-1-m)](q+j-1)^{m-j-1}}{[(j+1) \cdots m](q+j)(d-j)^{m}} \\
& \geq \frac{[3(d-j)]^{m}(q+j-1)^{m-j-1}}{[(j+1) \cdots m](q+j)(d-j)^{m}} \\
& \geq \frac{3^{m}}{(j+1)(j+2)} \\
& \geq \frac{3^{q+j-1}}{(j+1)(j+2)},
\end{aligned}
$$

leading to

$$
\sum_{j=2}^{d-1} S_{j}^{-1} \leq 3^{1-q} \sum_{j=2}^{d-1} \frac{(j+1)(j+2)}{3^{j}}=\frac{11 \cdot 3^{d}-6 d^{2}-24 d-27}{4 \cdot 3^{d+q-1}} \leq \frac{1}{3^{7}}
$$

It follows from $(5.16)-(5.18)$ that

$$
\sum_{j=0}^{d-1} S_{j}^{-1} \leq 1 / 4
$$

Consequently, by summing (5.15) over $j=0, \cdots, d-1$, we obtain the desired inequality (5.14), since $d \geq 1$. The proof is complete.

\section{REFERENCES}

[1] A. B. Andreev and R. D. Lazarov. Superconvergence of the gradient for quadratic triangular finite element methods. Numer. Methods for PDEs, 4:15-32, 1988. 
[2] J. H. Brandts and M. Kř̂́” žek. Gradient superconvergence on uniform simplicial partitions of polytopes. IMA J. Numer. Anal., 2003 (to appear).

[3] S. C. Brenner and L. R. Scott. The Mathematical Theory of Finite Element Methods. SpringerVerlag, 1996.

[4] C.-M. Chen. Superconvergent points of Galerkin's method for two point boundary value problems. Numer. Math. J. Chinese Univ., 1:73-79, 1979 (in Chinese).

[5] C.-M. Chen. Optimal points of stress for triangular linear elements. Numer. Math. J. Chinese Univ., 2:12-20, 1980 (in Chinese).

[6] C.-M. Chen. Optimal stress points in tetrahedral finite elements. Xiangtan Univ. J. Natural Sci., 3:16-24, 1980 (in Chinese).

[7] C. M. Chen and Y. Q. Huang. High Accuracy Theory of Finite Element Methods. Hunan Science Press, Hunan, China, 1995 (in Chinese).

[8] C.-M. Chen and Q.-D. Zhu. A new error estimate and theorm on optimal stress points in the finite element method. Xiangtan Univ. J. Natural Sci., 1, 1978 (in Chinese).

[9] P. G. Ciarlet. The finite Element Method for Elliptic Problems. North-Holland, Amsterdam, 1978.

[10] R. D. Dunlap. Superconvergence points in locally uniform finite element meshes for second order two-point boundary value problems. PhD thesis, Cornell University, 1996.

[11] H. Engles. Numerical Quadrature and Cubature. Academic Press, 1980.

[12] G. Goodsell. Pointwise superconvergence of the gradient for the linear tetrahedral element. Numer. Methods PDEs, 10:651-666, 1994.

[13] G. Goodsell and J. R. Whiteman. Superconvergence of recovered gradients of piecewise quadratic finite element approximations, Part I, $L^{2}$-error estimates. Numer. Methods PDEs, 7:6183, 1991.

[14] G. Goodsell and J. R. Whiteman. Superconvergence of recovered gradients of piecewise quadratic finite element approximations, Part II, $L^{\infty}$-error estimates. Numer. Methods PDEs, 7:8599, 1991.

[15] E. Isaacson and H. B. Keller. Analysis of Numerical Methods. Dover, 1994.

[16] J. Douglas Jr., T. Dupont, and M. F. Wheeler. An $L_{\infty}$ estimate and superconvergence result for a Galerkin method for elliptic equations based on tensor products of piecewise polynomials. RAIRO Modél. Math. Anal. Numér., 8:61-66, 1974.

[17] M. Kř́žek and P. Neittaanmäki. On superconvergence techniques. Acta Appl. Math., 9:175-198, 1987.

[18] P. Lesaint and M. Zlámal. Superconvergence of the gradient of finite element solutions. RAIRO Modél. Math. Anal. Numér., 13:139-166, 1979.

[19] N. D. Levine. Superconvergent recovery of the gradient from piecewise linear finite element approximations. IMA J. Numer. Anal., 5:407-427, 1985.

[20] B. Li. Superconvergence for higher-order triangular finite elements. Chinese J. Numer. Math. Appl., 12:75-79, 1990.

[21] Q. Lin and J. Xu. Linear finite elements with high accuracy. J. Comp. Math., 3:115-133, 1985.

[22] M. T. Nakao. Superconvergence of the gradient of Galerkin approximations for elliptic problems. RAIRO Modél. Math. Anal. Numér., 21:679-695, 1987.

[23] G. Szgo. Orthogonal Polynomials. Amer. Math. Soc., 3rd edition, 1967.

[24] L. B. Wahlbin. Superconvergence in Galerkin Finite Element Methods, volume 1605 of Lecture Notes in Mathematics. Springer, Berlin, 1995.

[25] Z. Zhang. Ultraconvergence of the patch recovery technique. Math. Comp., 65:1431-1437, 1996.

[26] Q.-D. Zhu. Optimal derivative points in the triangular quadratic finite elements. Xiangtan Univ. J. Natural Sci., 3:36-45, 1981 (in Chinese).

[27] Q.-D. Zhu. A superconvergence result for the finite element method. Numer. Math. J. Chinese Univ., 3:50-55, 1981 (in Chinese). 
[28] Q.-D. Zhu. Natural inner superconvergence for the finite element method. In K. Feng and J. L. Lions, editors, Proceedings of China-France Symposium on Finite Element Methods, pages 935960, Beijing, 1983. Gordon and Breach, New York.

[29] Q.-D. Zhu. A review of two different approaches for superconvergence analysis. Appl. Math., 43:401-411, 1998.

[30] Q. D. Zhu and Q. Lin. Superconvergence Theory of the Finite Element Method. Hunan Science Press, Hunan, China, 1989 (in Chinese).

Department of Mathematics, University of Maryland, College Park, MD 20742, U.S.A.

E-mail address: bli@math.umd.edu 